\title{
A New Approach to Designing the S-Shaped Annular Duct for Industrial Centrifugal Compressor
}

\author{
Ivan Yurko and German Bondarenko \\ Technical Thermophysics Department, Sumy State University, 2 Rimsky-Korsakov Street, Sumy 40007, Ukraine \\ Correspondence should be addressed to Ivan Yurko; yurkoi@mail.ru
}

Received 21 February 2014; Revised 11 July 2014; Accepted 20 August 2014; Published 16 October 2014

Academic Editor: Ting Wang

Copyright ( 2014 I. Yurko and G. Bondarenko. This is an open access article distributed under the Creative Commons Attribution License, which permits unrestricted use, distribution, and reproduction in any medium, provided the original work is properly cited.

The authors propose an analytical method for designing the inlet annular duct for an industrial centrifugal compressor using high-order Bezier curves. Using the design of experiments (DOE) theory, the three-level full factorial design was developed for determination of influence of the dimensionless geometric parameters on the output criteria. Numerical research was carried out for determination of pressure loss coefficients and velocity swirl angles using the software system ANSYS CFX. Optimal values of the slope for a wide range of geometric parameters, allowing minimizing losses in the duct, have been found. The study has used modern computational fluid dynamics techniques to develop a generalized technique for future development of efficient variable inlet guide vane systems. Recommendations for design of the s-shaped annular duct for industrial centrifugal compressor have been given.

\section{Introduction}

Most modern industrial centrifugal compressors are required to operate over a broad range of flows and pressure ratios whilst maintaining optimum efficiency.

To achieve this, it is common to use variable inlet guide vanes (VIGVs). Variable inlet guide vanes are used to generate inlet swirl in the flow upstream of impellers in order to reestablish optimum impeller incidence for variable mass flow rate at constant rotational speed.

According to the Euler momentum equation, changing of the circumferential velocity component of the inlet flow influences the energy transfer in the impeller:

$$
h_{t, 2}-h_{t, 1}=u_{2} c_{u 2}-u_{1} c_{u 1} \text {. }
$$

A reduction in mass flow rate in backward leaned impellers will lead to increasing of the exit swirl $\Delta c_{u 2}$, as shown in Figure 1.

This allows compensating for the change in inlet conditions and, depending on the impeller outlet angle, may even reduce the change in energy transfer to zero:

$$
\Delta\left(h_{t, 2}-h_{t, 1}\right)=u_{2} \Delta c_{u 2}-u_{1} \Delta c_{u 1} \approx 0,
$$

where $\Delta c_{u}=c_{u}{ }^{\prime}-c_{u}$. This property allows adjusting mass flow rate with a minimum change of total pressure ratio at a constant shaft speed. This extra control parameter is particularly useful for compressors with variable operating conditions, such as in process compressors, aeration treatment of sewage, air conditioning systems, and potentially in turbochargers [1-3]. Positive preswirl (in the direction of shaft rotation) allows maintaining optimum incidence at reduced mass flow, Figure 1. It is necessary to use large swirl angles to operate centrifugal compressor at low mass flow rates. The overall stage performance of industrial centrifugal compressors and an extension of the operating range can be improved using a high inlet swirl. The ensuing reduction of inlet Mach number has a favorable effect on performance. Hence, VIGVs are mostly used to produce positive preswirl in order to reduce the mass flow rate at constant pressure ratio.

The design of modern VIGV consists of two elements: vane cascade and annular flow duct connecting vane cascade and centrifugal impeller (Figure 2).

According to the conservation of angular momentum, the reduction of duct radius intensifies the induced swirl. It can lead to preswirl angles which exceed vane setting angles [4]. Therefore, it is possible to reduce a vane setting angle 


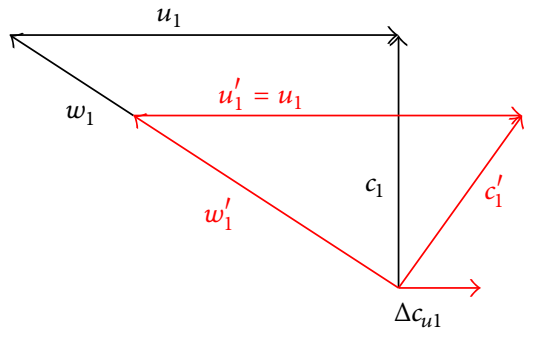

(a)

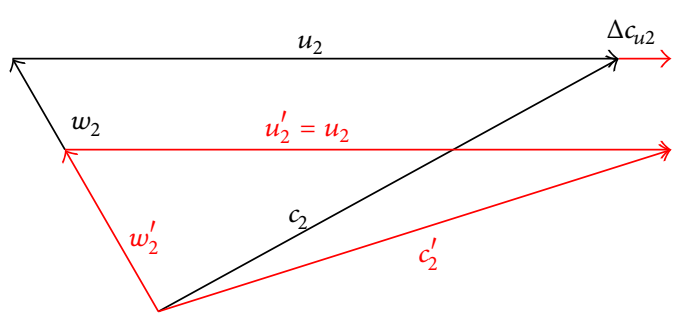

(b)

FIGURE 1: Velocity triangles for zero (black) and positive preswirl (red with primes) at different mass flow rates: (a) impeller inlet, (b) impeller exit.

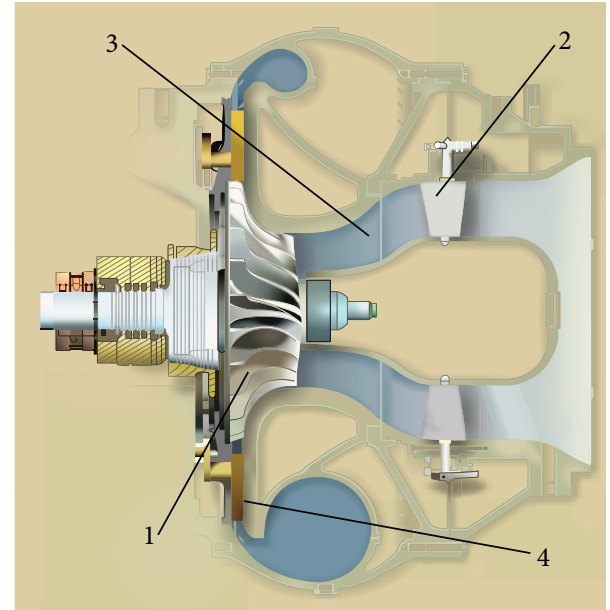

FIGURE 2: Modern design of centrifugal compressor with VIGV: 1 is impeller, 2 is vane cascade, 3 is annular duct, and 4 is diffuser.

that leads to essential decreasing of pressure loss coefficient. It can be used for providing a demanded flow angle. The main role of the annular duct is to increase swirl angle of the flow moving from the annular cascade, set on a larger radius, to the smaller radius, where the entrance to the impeller is located. Ideally, the IGV duct will be designed with a spherical section hub and shroud. The use of a spherical surface hub and shroud resulted in minimal constant blade clearance at the hub and shroud for any vane setting angle. If the hub and shroud are designed to be spherical sections, the duct through the IGV section must converge from the radius at the vanes to the radius of the impeller. Consequently, there is a need to design the s-shaped duct.

Bailey et al. [5] investigated the aerodynamic performance of a compressor s-shaped duct with a single strut. The data have shown that, as a result of flow curvature, significant streamwise pressure gradients exist within the duct, with this curvature also affecting the generation and suppression of turbulence. Also, the Blockage of strut was found to have a significant effect on pressure field of the duct, which has a direct influence on the turbulent flow field. Dueñas et al. [6] had experimentally investigated the effect of reducing the duct length on its performance, keeping duct inlet height $\left(h_{\text {in }}\right)$ and inlet to exit radius change $(\Delta r)$. As the duct length was reduced the loss increases mainly due to boundary layer separation. It was found that the length of original duct without strut reducing to $74 \%$ caused a small rise in loss. However, reducing the length to $64 \%$ caused a much larger rise in loss. The researches have shown that the limit of the design space of annular s-ducts is set by duct corner separation.

In order to reduce the extent of the corner separation and avoid higher loss coefficient in ducts, the focus lies on intermediate s-shaped duct endwall profiling and its influence on the flow field in the duct.

A number of works have been carried out as part of the EU research project AIDA (Aggressive Intermediate Duct Aerodynamics for Competitive and Environmentally Friendly Jet Engines), Contract no. AST3-CT-2003-502836. In these papers emphasis is placed on the overall performance of duct geometry typical of that which may be found in a modern multispool gas turbine engine. Several parameters have been used by authors to quantify, geometrically, the aggressiveness of an s-shaped duct: nondimensional radius change $(\Delta r / L)$, duct area ratio $\left(A_{0} / A_{1}\right)$, and nondimensional length $\left(L / h_{\text {in }}\right)$. In these papers the design and performance evaluating of an intermediate turbine duct for different operating conditions have been presented [7, 8]. Marn et al. [9] have carried out a detailed experimental investigation of the flow field development and its implications for the turbine duct performance. Walker et al. [10] have shown that the flow characteristics are very dependent on the duct geometrical parameters.

However, the most important difference lies in the fact that all these studies are related to turbine ducts. The main difference between the compressor duct and turbine duct consists in a flow direction. Intermediate turbine ducts are used for connecting the high pressure turbine at smaller diameters with the low-pressure turbine at larger diameters. Therefore, turbine duct in most cases is made with much diffusion. However, much diffusion may negatively influence flow in s-shaped compressor duct.

Only a limited amount of published data is available and this information may be of more immediate use to the centrifugal compressor.

Swain [11] proposed basic rules that govern the design of the inlet s-shaped duct geometry for centrifugal compressor. 
However, a set of these rules rigidly link duct length with setting radius. With a large setting radius, the duct has a large bend in the hub surface, which contributes significantly to duct losses. Besides, sometimes it is required to set the concrete duct length regardless of other chosen parameters.

The inlet duct shares an equal proportion of the total system losses with vanes at high setting angles. The duct shape also affects the magnitude and distribution of velocities and swirl angles at the impeller inlet.

The main aim of the proposed paper is to create a methodology for designing highly efficient annular flow duct for centrifugal compressors, which provides minimal losses over the entire range of vane setting angle. An additional aim is study of influence of the annular flow duct main geometrical parameters on the magnitude of losses and swirl angles in it, using the theory of design of experiments (DOE) and subsequent optimization of the duct shape.

\section{Proposed Design Method}

The authors of this paper propose that the inlet duct shape could be defined by two main and two additional parameters. The main parameters are the radius ratio $\overline{R_{r}}$ and the area ratio $\overline{A_{r}}$. Additional parameters are the slope $\bar{Y}$ and plane of inflection $\bar{S}$ (see Figure 3). These parameters are defined as

radius ratio:

$$
\overline{R_{r}}=\frac{R_{S 0}+R_{H 0}}{R_{S 1}+R_{H 1}}
$$

area ratio:

$$
\overline{A_{r}}=\frac{R_{S 0}^{2}-R_{H 0}^{2}}{R_{S 1}^{2}-R_{H 1}^{2}}
$$

where $R_{S 1}$ and $R_{H 1}$ are dictated by the impeller hub and shroud radiuses (these sizes are known from the geometry of the impeller). Simultaneously solving equations for $\overline{R_{r}}(3)$ and $\overline{A_{r}}(4)$, variables $R_{H 0}$ and $R_{S 0}$ can be found.

Slope is

$$
\begin{aligned}
\bar{Y}=\tan \gamma & =\frac{\left(R_{S 0}+R_{H 0}\right)-\left(R_{S 1}+R_{H 1}\right)}{2 L} \\
& =\frac{\left(\overline{R_{r}}-1\right)\left(R_{S 1}+R_{H 1}\right)}{2 L} .
\end{aligned}
$$

Plane of inflection is

$$
\bar{S}=\frac{x}{L} .
$$

Shape of the hub and shroud contours of the duct were set using Bezier curves. The main advantages of these curves are easy parametrization of contours, smooth curvature change, and the possibility of creating meridional contours of any shape using the minimum number of control points. Bezier

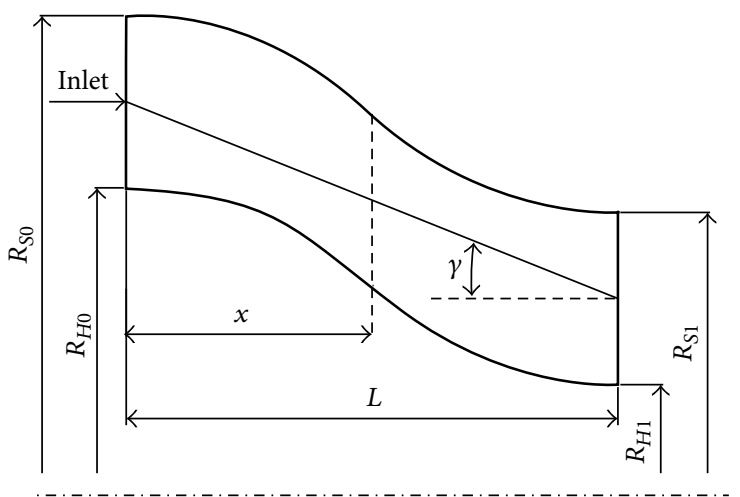

FIgURE 3: Parametric s-shaped annular flow duct geometry.

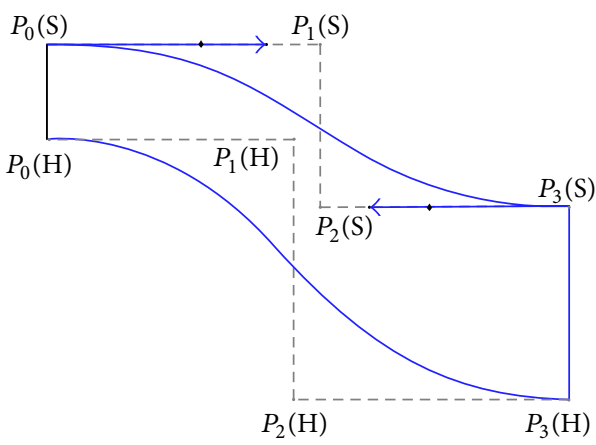

FIgURE 4: Control points of Bezier curves.

curve is a parametric curve, which has the following formula for the general form:

$$
B(t)=\sum_{i=0}^{n} P_{i} \cdot b_{i, n}(t),
$$

where $0 \leq t \leq 1$ is dimensionless length along the Bezier curve. $P_{i}$ is a function of the coordinates of supporting vertices; $b_{i, n}(t)=(n ! /(i !(n-i) !)) t^{i}(1-t)^{n-i}$ is Bernstein basis function, where $n$ is the order of the Bezier curve.

Meridional contours of the duct have an s-shape, so they need to use Bezier curve of the third order for their creation (cubic Bezier curves). To completely define the shape of the Bezier curve, it is just enough coordinates for 4 points: two, which correspond to coordinates of the beginning and end of the curve, and remaining points, which correspond to shape for bending. Inlet annular duct consists of hub and shroud contours and therefore of 8 control points (see Figure 4).

The equations $x=\varphi(t)$ and $y=\psi(t)$ establish dependency between the Cartesian coordinates $(x, y)$ and value of the parameter $t$ and define the cubic curve coordinates by the following equations:

$$
\begin{aligned}
& x(t)=(1-t)^{3} x_{0}+3 t(1-t)^{2} x_{1}+3 t^{2}(1-t) x_{2}+t^{3} x_{3}, \\
& y(t)=(1-t)^{3} y_{0}+3 t(1-t)^{2} y_{1}+3 t^{2}(1-t) y_{2}+t^{3} y_{3} .
\end{aligned}
$$

A number of rules for reduction of quantity of controlled points were accepted. 


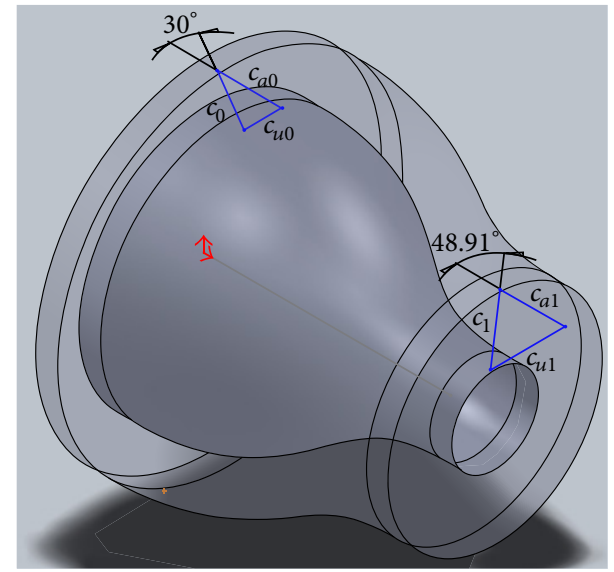

Figure 5: Three-dimensional model of the inlet annular duct with velocity triangles.

(1) Coordinates of points $P_{3 S}\left[x_{3 S}, y_{3 S}\right]$ and $P_{3 H}\left[x_{3 H}, y_{3 H}\right]$ are known from the geometry of the impeller.

(2) Coordinates of points $P_{0 S}\left[x_{0 S}, y_{0 S}\right], P_{0 H}\left[x_{0 H}, y_{0 H}\right]$, $P_{3 S}\left[x_{3 S}, y_{3 S}\right]$, and $P_{3 H}\left[x_{3 H}, y_{3 H}\right]$ are defined from the equations for $\overline{A_{r}}, \overline{R_{r}}$, and $\bar{Y}: y_{0 S}=R_{S 0}, y_{0 H}=R_{H 0}$, $y_{3 S}=R_{S 1}, y_{3 H}=R_{H 1} \cdot x_{0 H}=x_{0 S}=0$, and $x_{3 H}=$ $x_{3 S}=L$ (see Figures 3 and 4$)$.

(3) The beginning and end of Bezier curves are located tangentially to inlet and outlet of the annular duct; therefore, $y_{0 S}=y_{1 S}, y_{0 H}=y_{1 H}, y_{2 S}=y_{3 S}$, and $y_{2 H}=y_{3 H}$.

(4) Control points $P_{1 S}, P_{2 S}, P_{1 H}$, and $P_{2 H}$ are located on the same vertical axis $\left(x_{1 H}=x_{1 S}=x_{2 H}=x_{2 S}\right)$ and form the plane of inflection.

A total of 16 coordinates $x$ and $y$ were replaced by four dimensionless parameters that allow designing the annular duct of any desired shape.

The program for designing the annular duct of any shape in automatic mode has been developed. An example of the designed duct is presented in Figure 5. Later, this geometrical model was exported to the numerical solver.

The problem of optimization consists in search of such admissible combination of factors, satisfying the constraints and obtaining extreme value of the objective function.

But at first, it is necessary to define influence of each factor separately on objective function. To solve this problem, the DOE and regression theory was used.

The second-order polynomial was chosen as functional relationship between dimensionless parameters of the duct and the output criteria [12]:

$$
y=a_{0}+\sum_{i=1}^{n} a_{i} x_{i}+\sum_{i=1}^{n} a_{i i} x_{i}^{2}+\sum_{i=1}^{n} \sum_{j<i} a_{i j} x_{i} x_{j}
$$

where $x_{i}$ are input factors and $a_{i}$ are coefficients of polynomial.

For determining regression coefficients of the polynomial (9), the three-level full factorial design was developed.
TABLE 1: Matrix of the $3^{3}$ design.

\begin{tabular}{lccc}
\hline Input factors & Low-level $(-1)$ & Mid-level $(0)$ & High-level $(+1)$ \\
\hline$\overline{R_{r}}$ & 1.3 & 1.9 & 2.5 \\
$\overline{A_{r}}$ & 1 & 1.5 & 2 \\
$\bar{Y}$ & 0.2 & 0.5 & 0.8 \\
\hline
\end{tabular}

These three-level designs have been used to model possible curvature in the response function. The third level for the continuous factor facilitates investigation of a quadratic relationship between the response and each of the factors.

Pressure loss coefficient and swirl angle at the outlet were set as response variables.

Through conservation of angular momentum and continuity, the theoretical swirl angle $\alpha_{1}$ (see Figure 6) at the centrifugal impeller inlet could be predicted on the basis of the vane setting angle, the radius ratio, and the area ratio:

$$
\begin{aligned}
c_{u 0} r_{0} & =c_{u 1} r_{1}, \\
\rho_{0} \cdot c_{a 0} \cdot F_{0} & =\rho_{1} \cdot c_{a 1} \cdot F_{1} .
\end{aligned}
$$

Since $\tan \alpha_{0}=\left(c_{u 0} / c_{a 0}\right)$ (see Figure 6)

$$
\begin{gathered}
\rho_{0} \cdot c_{a 0} \cdot F_{0}=\rho_{1} \cdot c_{a 1} \cdot F_{1}, \\
c_{a 0} \cdot \tan \alpha_{0} \cdot r_{0}=c_{a 1} \cdot \tan \alpha_{1} \cdot r_{1} .
\end{gathered}
$$

By dividing the first equation to the second $\left(\rho_{0} \cdot F_{0}\right) /\left(\tan \alpha_{0}\right.$. $\left.r_{0}\right)=\left(\rho_{1} \cdot F_{1}\right) /\left(\tan \alpha_{1} \cdot r_{1}\right)$, we obtain the result

$$
\tan \alpha_{1}=\tan \alpha_{0} \cdot \frac{\rho_{1}}{\rho_{0}} \cdot \frac{F_{1}}{F_{0}} \cdot \frac{r_{0}}{r_{1}}=\tan \alpha_{0} \cdot \frac{\rho_{1}}{\rho_{0}} \cdot \frac{\overline{R_{r}}}{\overline{A_{r}}} .
$$

A high radius ratio would tend to increase the turning due to conservation of angular momentum. However, larger area ratios have the effect of accelerating the axial component of the flow at the impeller inlet and reducing the magnitude of the swirl angle due to continuity (Figure 6).

The trends exhibited by these suggest that an ideal system would possess a high radius ratio and a low area ratio. Density at the exit of the duct will be lower than at the inlet, so real swirl angle will also be smaller, compared to noncompressible flow.

Final range of the settings for input factors is shown in Table 1.

Inflection plane was located at the middle of the duct length, which corresponds to the parameter $\bar{S}=x / L=0.5$ in all numerical calculations. Swirl angle at the inlet $\alpha_{0}$ was set as a categorical variable. Calculations were performed for the following angles: $0^{\circ}, 15^{\circ}, 30^{\circ}$, and $45^{\circ}$ (DOE matrix was the same for each swirl angle). The number of runs required to estimate main terms present in a three-factor quadratic model is 27 per each swirl angle at the inlet. As a result 108 calculations have been carried out.

\section{Numerical Simulation}

The performance of annular duct at different geometries and swirl angles was analyzed by means of "Ansys CFX." In addition to accurate geometrical representation and grid quality, 


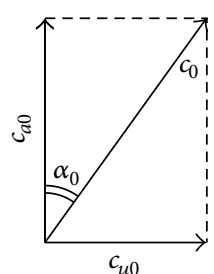

(a)

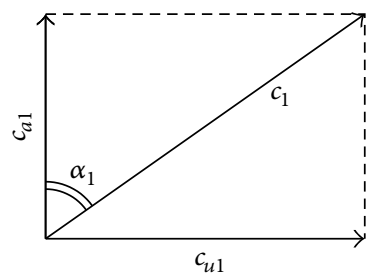

(b)

FIGURE 6: Velocity triangles at inlet (a) and outlet (b) of the annular duct.

the grid resolution is another important factor in practical CFD simulations. If too few mesh cells are used in the simulation, the accuracy of the results may be compromised. It is therefore usual to conduct a grid dependency study at the outset of the CFD analyses to ensure that an optimum grid resolution is used throughout. Computational mesh consists of unstructured tetrahedral cells and prismatic layer cells for boundary layer resolution. Value of dimensionless wall distance $y^{+}$is less than 2 . The quantity of cells within the boundary layers was equal to about 20. Compliance with these requirements is necessary for the correct application of the SST turbulence model proposed by Menter [13].

During the simulation, the issue of grid independence results was separately investigated.

A grid dependency study has been performed according to requirements [14]. Several variants of computational grid were considered: "coarse," consisting of 14000 elements, "medium," consisting of 37000 elements, "fine," consisting of 120000 elements, and "finest," consisting of 180000 elements. Grid-independency solution was performed for undesired duct geometry which caused flow separation. Calculation was carried out for three different convection schemes: first-order upwind scheme, high-resolution scheme, and second-order scheme (specified blend factor $=1$ ).

Comparing the results of the integral characteristics of losses, a grid density of approximately 120000 cells (cell size approximately $0.5 \mathrm{~mm}$ ) was therefore considered to provide sufficient accuracy and resolution to be adopted as the standard for all future working grids (see Figure $7(\mathrm{c})$ ). This number of cells provides a compromise between the accuracy and the calculation time.

The steady-state model and a high-resolution scheme with the specified blend factor value of 1 that is second-order accurate in space were used to solve the convection-diffusion equations.

Due to the circumferential periodicity of most turbomachines, only one sector needs to be modeled since periodic boundary conditions can be applied to the sides of the domain. Material is air ideal gas. Heat transfer model is set to total energy. Computations were made with a uniform total pressure, temperature, and flow direction (swirl angle) at the inlet. Mass flow rate was used as outlet conditions. Mass-average outlet swirl angle and the system total pressure loss coefficient were two main aerodynamic performance parameters which were used for comparison within the study, both of which should be unaffected by varying mass flow rate.
This assumption was confirmed by test simulations. As the convergence criteria, the discrepancy in total pressure loss coefficient equal to 0.001 has been used.

\section{Simulation Results}

Processing of the computer simulation results was performed using the programs Microsoft Excel and Statsoft Statistica. The validation of formal macromodel for adequacy showed that the relative error of output parameters in reference points was less than $1 \%$. It allows with a sufficient accuracy for engineering calculations to define output criteria in intermediate points.

It is important to determine the influence of dimensionless parameters on the output criteria for $\alpha_{0}=0^{\circ}$ (axial flow in the duct), because most of the time gas flows at this regime.

Let us consider the cases at the maximum value $\overline{R_{r}}=$ 2.5 and at the minimum value $\overline{R_{r}}=1.3$. Figure 8 illustrates the response surfaces, depending on area ratio $\overline{A_{r}}$, slope $\bar{Y}$, and output criteria $\zeta$. It can be seen from Figure 8 that the minimum losses are reached at the minimum value of the factor $\overline{A_{r}}$ for any slope $\bar{Y}$. Input factor $\bar{Y}$ has an optimum value which minimizes the response function for each $\overline{A_{r}}$. The difference between the losses at $\overline{A_{r}}=1$ and $\overline{A_{r}}=2$ for the case of the optimal slope reaches about $200 \%$. It can be concluded that it is necessary to decrease the parameter $\overline{A_{r}}$ for minimizing losses in the case of axial flow.

Increasing of the parameter $\overline{A_{r}}$ also influences negatively the losses in the presence of swirl flow in the duct (Figure 9(a)). As it was supposed earlier, the increasing of the $\overline{A_{r}}$ parameter reduces an efficient swirl angle $\alpha_{1}$ at the duct outlet (Figure 9(b)). Slope practically does not affect the swirl angle $\alpha_{1}$.

Figure 10(a) shows that the optimum value of the slope decreases with the decreasing $\overline{R_{r}}$ parameter. Optimal value of the slope means such a value at which the total pressure loss coefficient in the duct is minimal. Practically, it does not make sense to create the duct with the parameter $\overline{R_{r}}$ more than 2.5 as losses grow significantly, and the value of swirl angle almost does not increase (Figure 10(b)). Figure 11 shows velocity streamlines of axial flow in the duct at different slope $\bar{Y}$ parameters.

Separation zone is formed on the hub surface with a large slope. The more slope, the more separated zone. Therefore, 


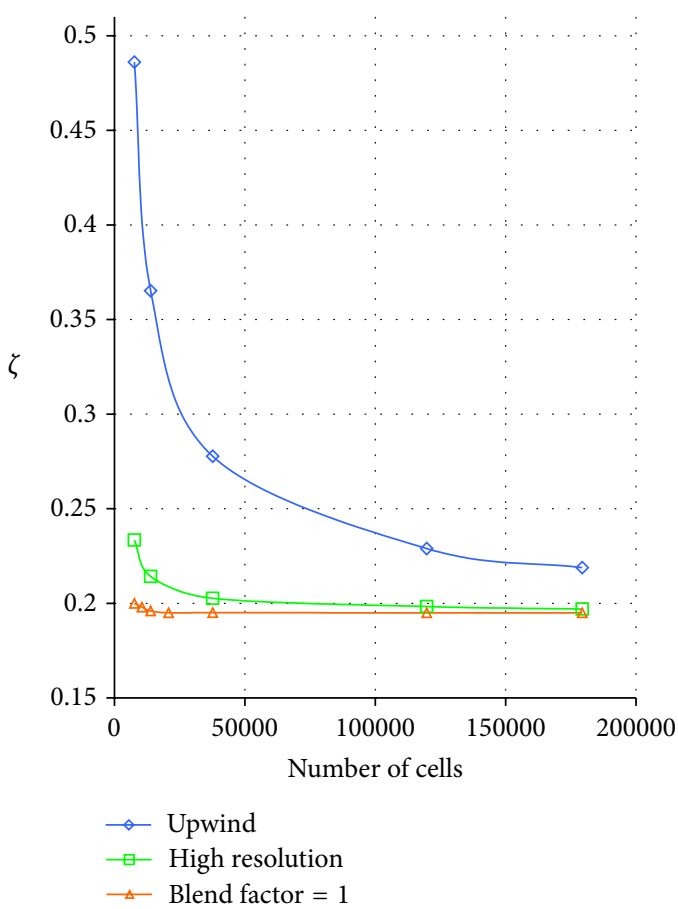

(a)

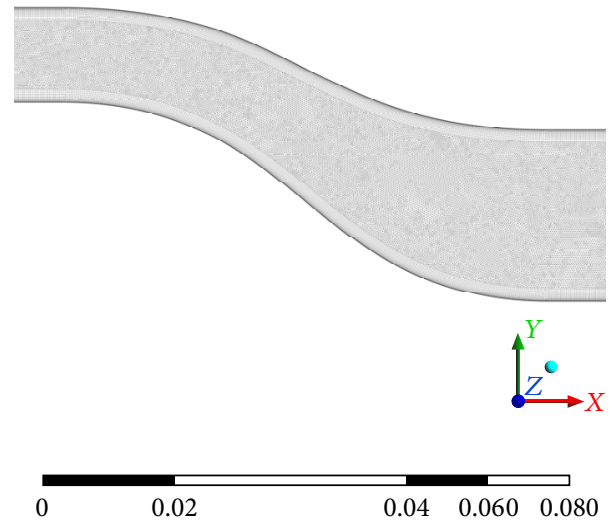

(m)

(b)

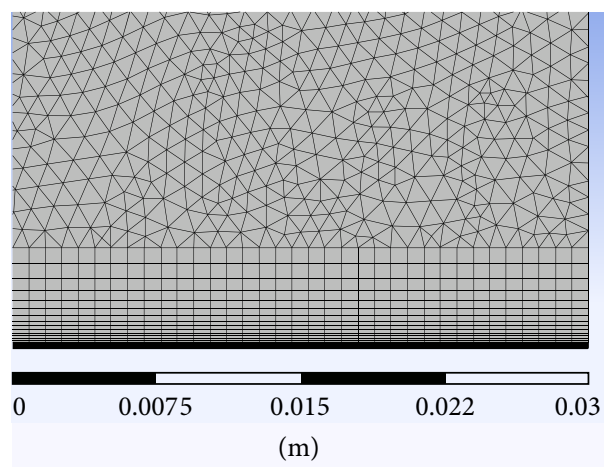

(c)

(c)

FIGURE 7: Annular duct mesh model: (a) grid convergence study, (b) CFD grid model, and (c) increased resolution of the grid at the exit.

it is necessary to find the optimum value of the slope, at which these separation zones will disappear. Pressure loss coefficient was chosen as a criterion of optimality. Experience shows that the minimum pressure loss coefficient in the duct is equivalent to state, when separation does not occur. Figure 12(a) shows the optimal values of the slope, which were calculated for different values of the $\overline{R_{r}}$ and inlet swirl angles $\alpha_{0}$. Figure 12(b) shows total pressure loss coefficient $\zeta$ at these optimal slopes. The law of losses dependence from the parameter $\overline{R_{r}}$ is different for each swirl angle $\alpha$. As it was mentioned earlier, the value of the optimal slope increases with the parameters $\overline{R_{r}}$ and $\alpha$ so the duct becomes steeper. Optimal meridional duct length can be determined from (5), based on $\bar{R}_{r}$ and corresponding $\bar{Y}_{\text {OPT }}$ found from Figure 12(a). The lower value of parameter $\overline{R_{r}}$ and the higher value of inlet swirl angle, the shorter duct length.
With swirling flow in the ducts, the separation on the hub surface will not occur. This is due to the fact that the swirling flow flows along the helical streamlines and actual path, which overcomes the flow, is much greater than the meridional duct length.

Figure 13 shows dependencies of $\alpha_{1}$ and $\zeta$ from the inlet swirl angle $\alpha_{0}$ for several values of parameter $\overline{R_{r}}$. This graph is plotted at value $\overline{A_{r}}=1$ and optimal slope $\bar{Y}_{\mathrm{OPT}}$ for each $\overline{R_{r}}$. For illustration purposes, the variable " $90^{\circ}-\alpha$ " was accepted instead of " $\alpha$ " for $Y$ axis on the right side.

Losses increase dramatically with the inlet swirl and $\overline{R_{r}}$ parameter. It can be explained because of the effective duct length which is increasing with swirl angle.

It is obvious that the minimum losses were achieved with minimum $\overline{R_{r}}$. But with decreasing $\overline{R_{r}}$ swirl angle $\alpha_{1}$ will be less, and therefore it will be necessary to increase the vane 


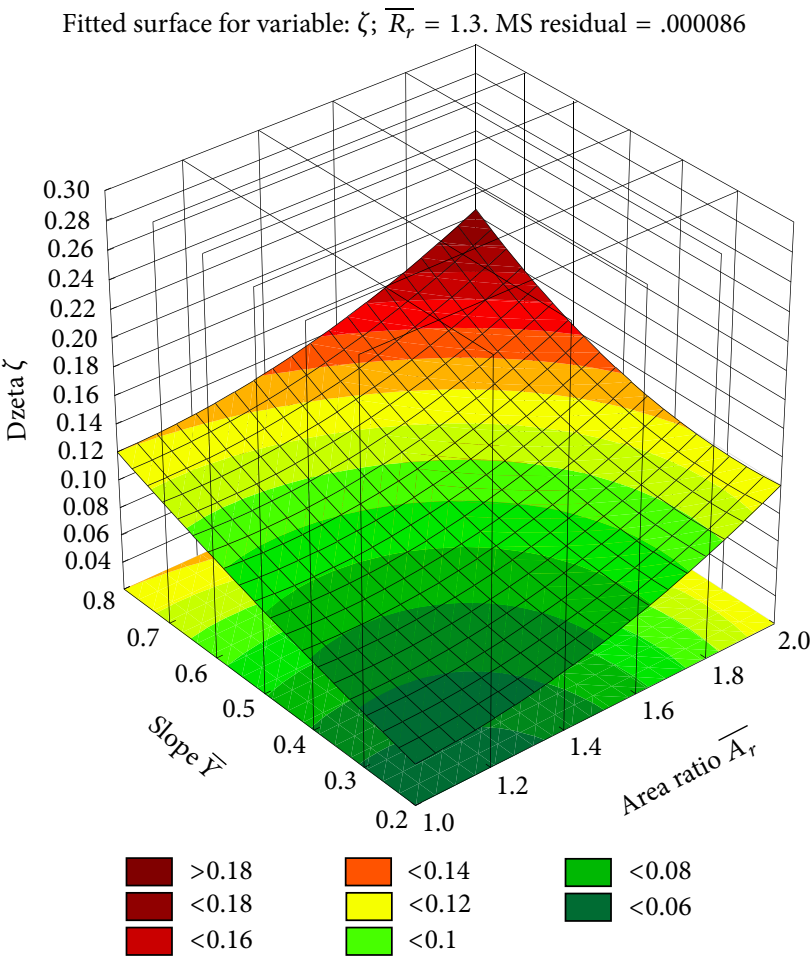

(a)

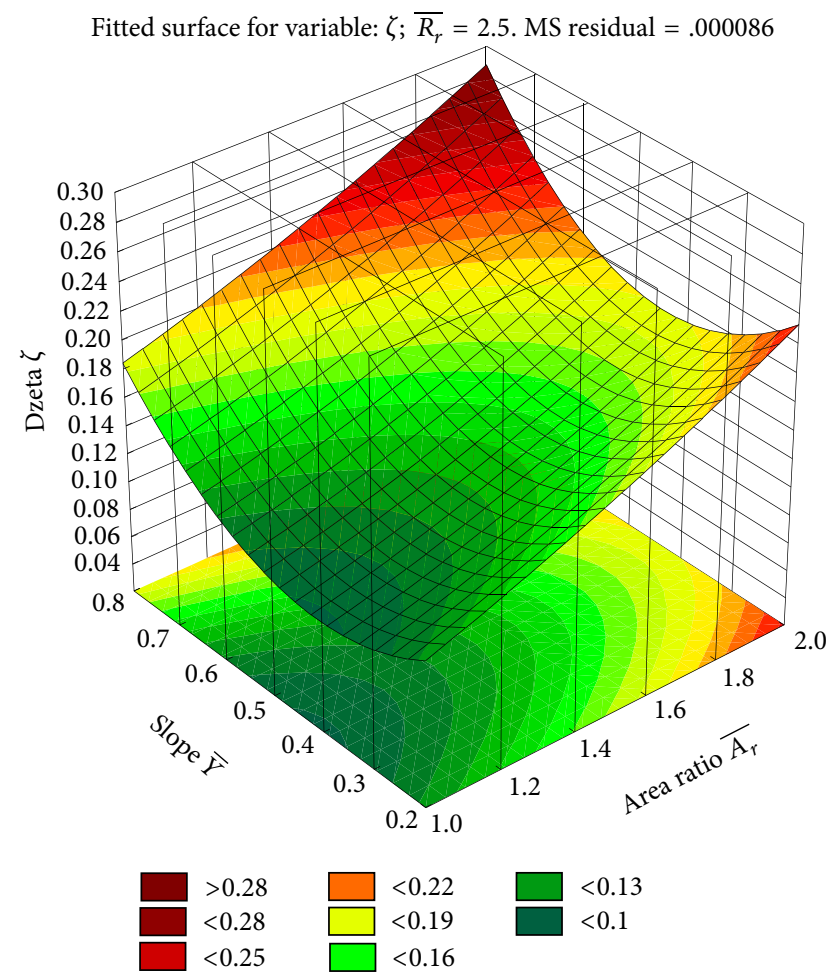

(b)

Figure 8: Response surface of output criteria $\zeta$ at $\overline{R_{r}}=1.3$ (a) and $\overline{R_{r}}=2.5$ (b) for $\alpha_{0}=0^{\circ}$.

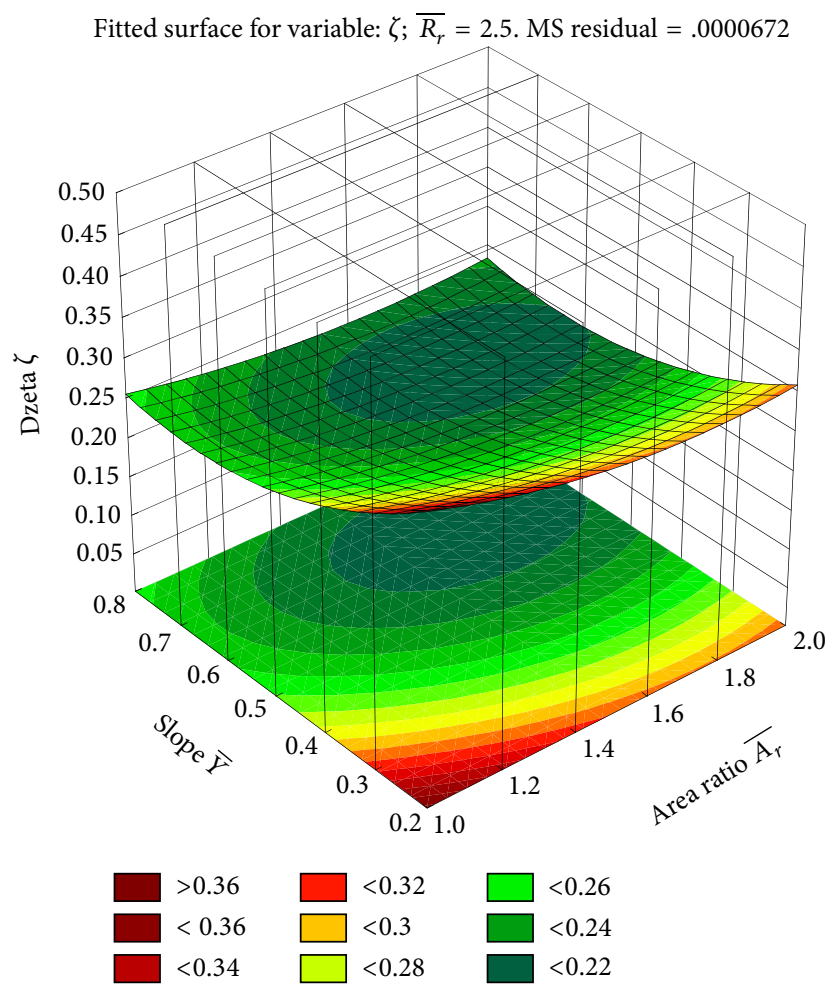

(a)

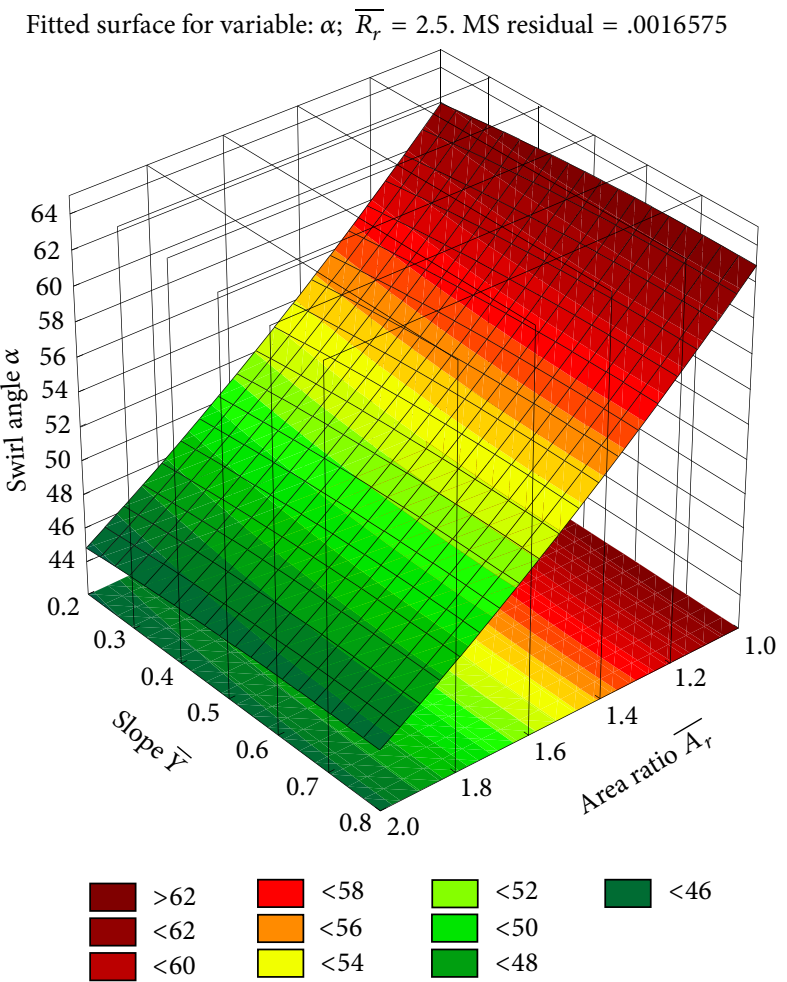

(b)

FIGURE 9: Response surfaces of output criteria $\zeta$ (a) and $\alpha_{1}$ (b) at $\overline{R_{r}}=2.5$ for $\alpha_{0}=45^{\circ}$. 


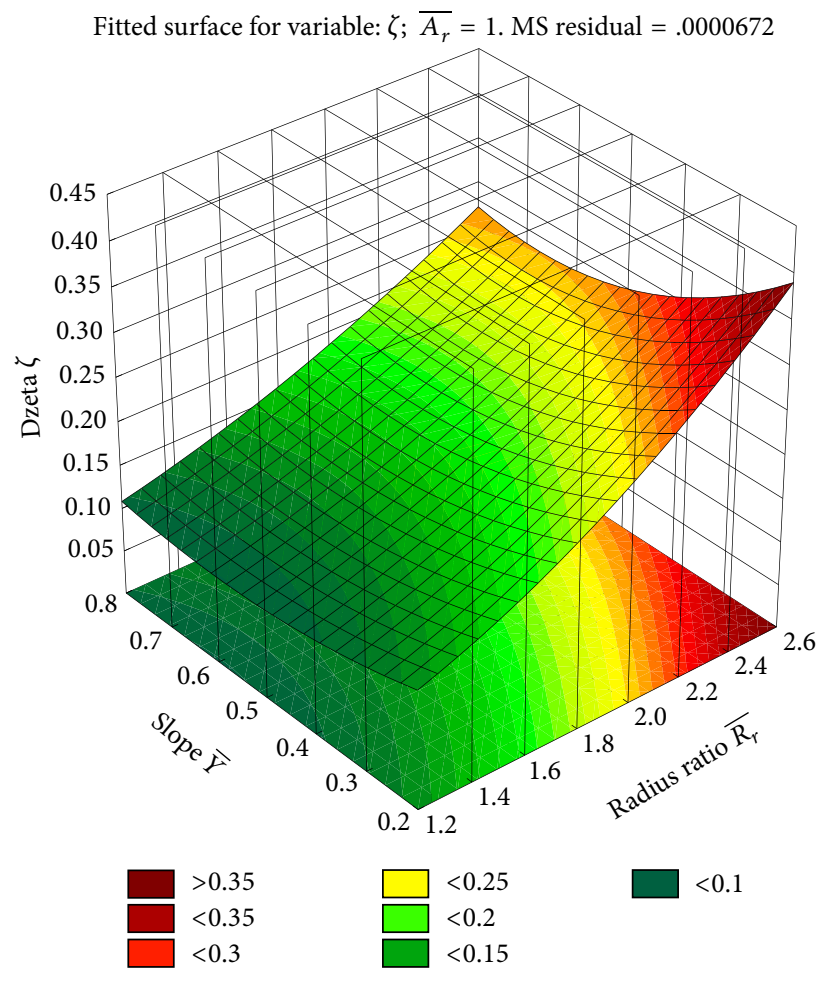

(a)

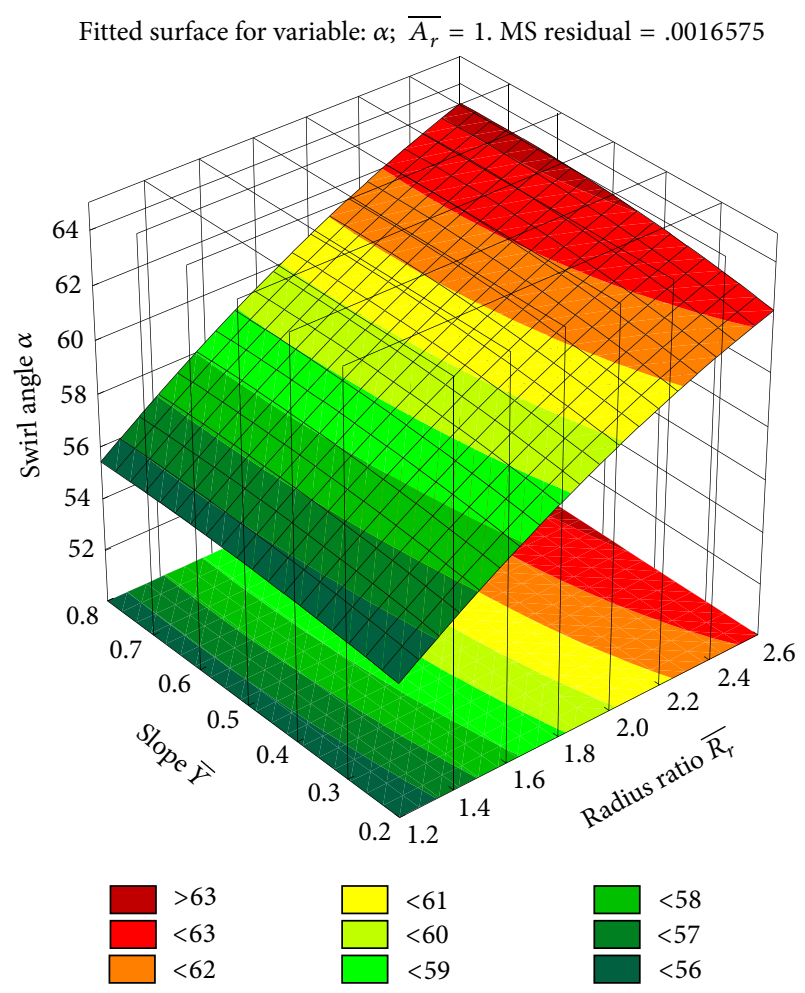

(b)

FiguRE 10: Response surfaces of output criteria $\zeta$ (a) and $\alpha$ (b) at $\overline{A_{r}}=1$ for $\alpha_{0}=45^{\circ}$.

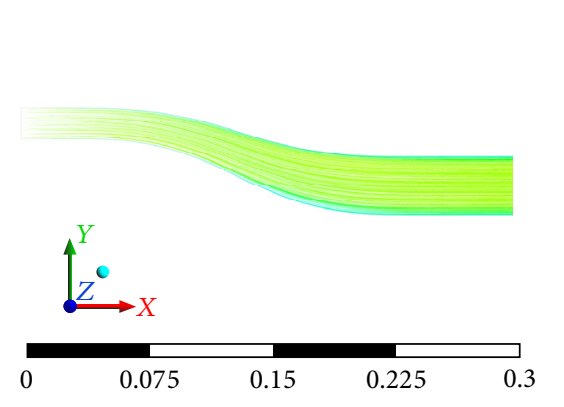

(m)

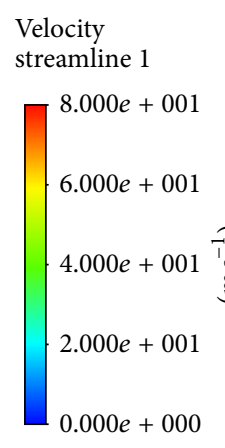

(a)

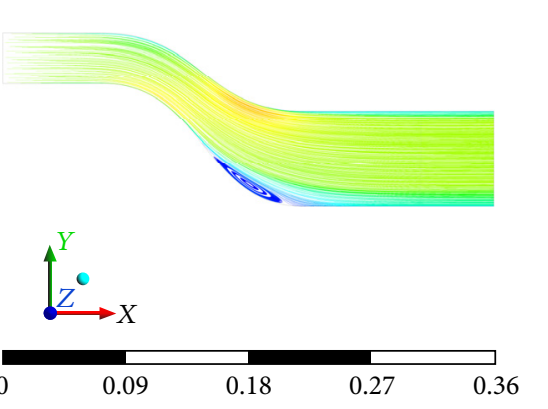

(m)

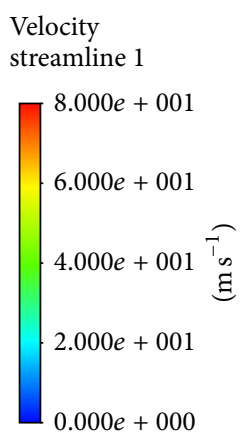

(b)

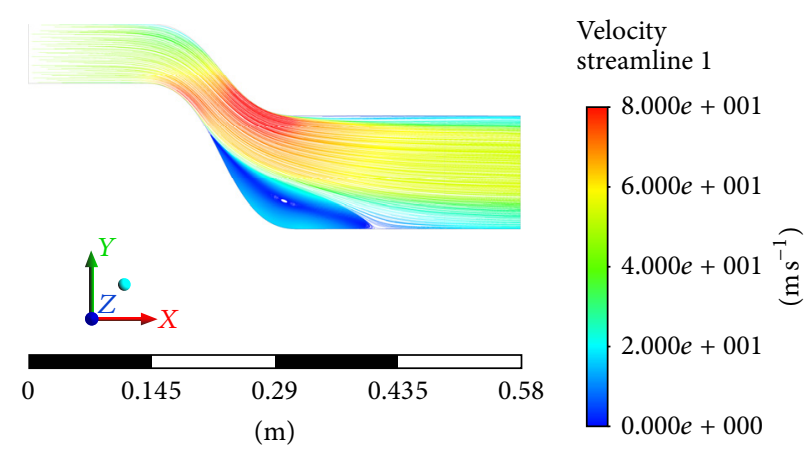

(c)

FIGURE 11: Velocity streamlines at $\overline{R_{r}}=1.9$ and $\alpha_{0}=0^{\circ}$ for $\bar{Y}=0.2$ (a), $\bar{Y}=0.5$ (b), and $\bar{Y}=0.8$ (c). 


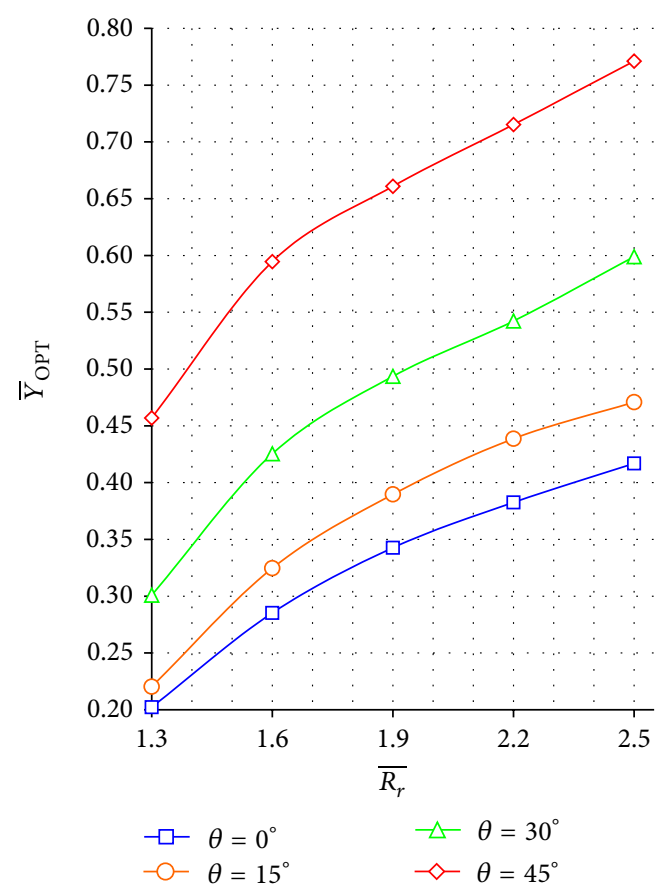

(a)

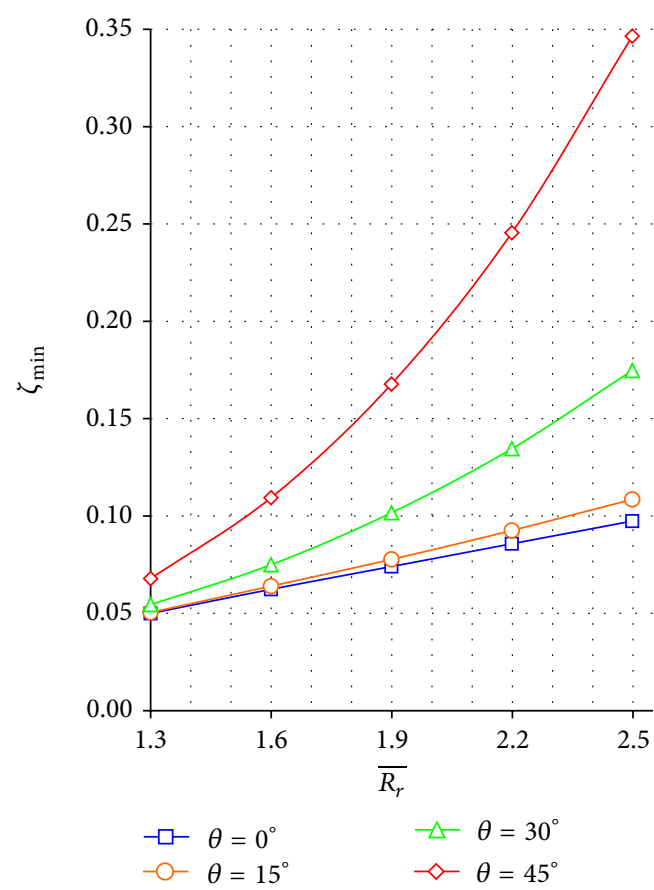

(b)

Figure 12: Charts of the optimal slope (a) and minimum losses $\zeta$, (b) for wide ranges of $\overline{R_{r}}$ and $\alpha$.

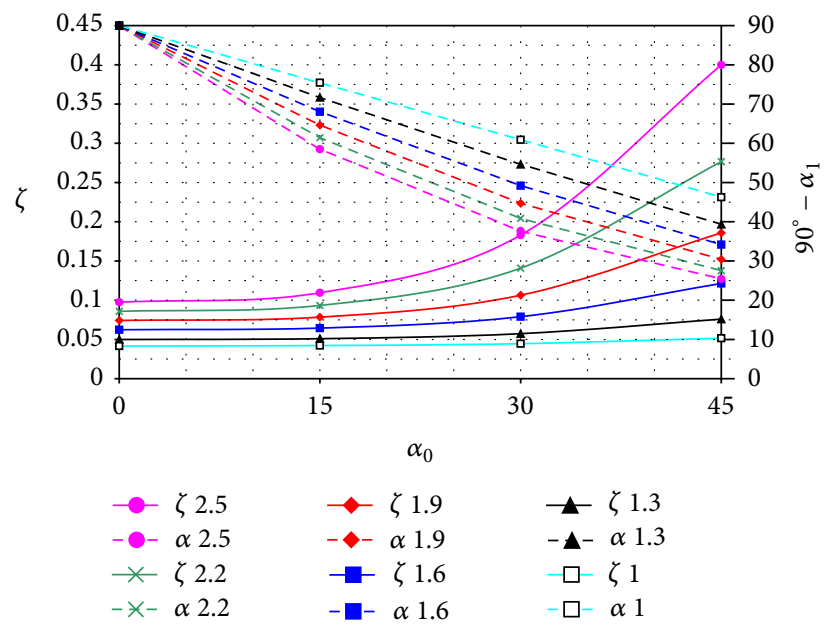

FIGURE 13: Graphs of $\alpha_{1}$ and $\zeta$ depending on the inlet swirl angles $\alpha_{0}$ at different $\overline{R_{r}}$.

setting angle to provide the required flow angle at the outlet from the annular duct. It will lead to an additional increase in losses in the vanes cascade. It turns out that the benefits of decreasing incidence onto vanes should cover the losses associated with the duct rise for a higher radius.

Therefore, it is necessary to know the losses in vane cascade for the final choice of the parameter $\overline{R_{r}}$. It will be done in a future research investigation. In general, the design of the annular duct seems to be robust and to fulfill its requirements as a baseline compressor's duct for further investigations.

\section{Conclusions}

Computational studies on the s-shaped duct are conducted. As conclusions, we would like to give the following recommendations for the design of the s-shaped annular duct for a centrifugal compressor.

(1) Inflection plane should be selected based on providing a minimum clearance between the vanes and the meridional contours. Additionally calculations showed that losses and swirl angle are increased in case of shifting the inflection plane in the direction of duct inlet.

(2) Designing ducts with the parameter greater than $\overline{R_{r}}>2.5$ does not make sense as the loss coefficient increases significantly, and the swirl angle practically does not increase.

(3) If the parameter $\overline{A_{r}}$ increases, the total pressure loss coefficient also increases and the outlet swirl angle decreases. Therefore, it is necessary to design the duct with the same area of the inlet and outlet $\left(\overline{A_{r}}=1\right)$ for increasing the output swirl angle. In some cases for additional increasing of swirl angle, there may be a slight decrease of parameter $\overline{A_{r}}$ lower than 1 .

(4) Duct length should be chosen on the optimal values of the slope parameter. Value of the optimal slope increases with increasing $\overline{R_{r}}$. 


\section{Nomenclature}

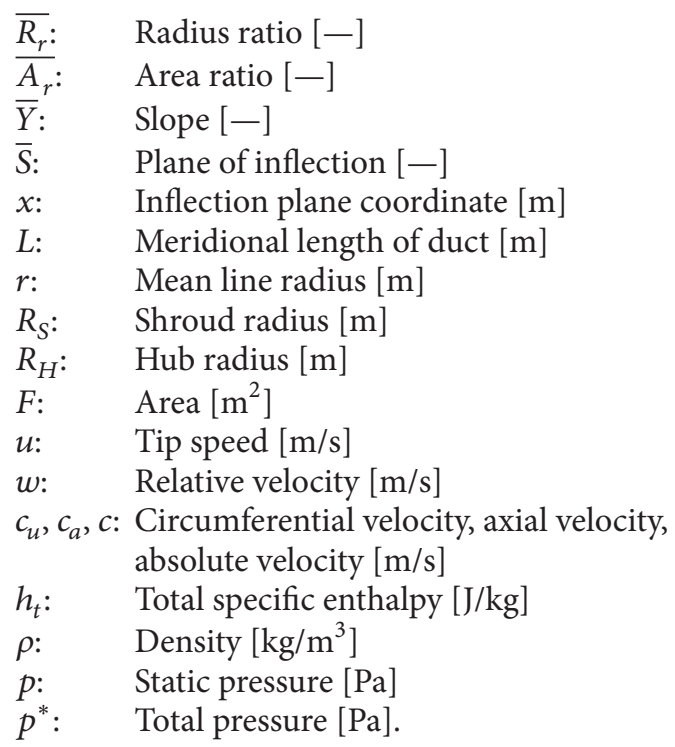

\section{Greek Symbols}

$\alpha$ (alfa): Swirl angle [ $\left.{ }^{\circ}\right], \alpha=\tan ^{-1}\left(c_{u 1} / c_{a 1}\right)$

$\zeta$ (dzeta): Total pressure loss coefficient [-], $\zeta=\left(p_{0}{ }^{*}-p_{1}{ }^{*}\right) /\left(p_{0}{ }^{*}-p_{0}\right)$.

\section{Subscripts}

0: Annular duct inlet

1: Annular duct outlet (impeller inlet)

2: Impeller outlet

$s$ : Shroud

$h$ : Hub.

\section{Superscripts}

': Parameters with positive preswirl.

\section{Conflict of Interests}

The authors declare that there is no conflict of interests regarding the publication of this paper.

\section{References}

[1] H. Simon, T. Wallmann, and T. Moenk, "Improvements in performance characteristics of single-stage and multistage centrifugal compressors by simultaneous adjustments of inlet guide vanes and diffuser vanes," Journal of Turbomachinery, vol. 109, no. 1, pp. 41-47, 1987.

[2] C. Rodgers, "Centrifugal compressor inlet guide vanes for increased surge margin," Journal of Turbomachinery, vol. 113, no. 4, pp. 696-702, 1991.

[3] A. Whitfield and A. H. Abdullah, "The performance of a centrifugal compressor with high inlet prewhirl," Journal of Turbomachinery, vol. 120, no. 3, pp. 487-493, 1998.
[4] M. Coppinger and E. Swain, "Performance prediction of an industrial centrifugal compressor inlet guide vane system," Proceedings of the Institution of Mechanical Engineers A: Journal of Power and Energy, vol. 214, no. 2, pp. 153-164, 2000.

[5] D. W. Bailey, K. M. Britchford, J. F. Carrotte, and S. J. Stevens, "Performance assessment of an annular S-shaped duct," Journal of Turbomachinery, vol. 119, no. 1, pp. 149-156, 1997.

[6] C. O. Dueñas, R. J. Miller, H. P. Hodson, and J. P. Longley, "Effect of length on compressor inter-stage duct performance," in Proceedings of the ASME Turbo Expo, paper no. GT200727752, pp. 319-329, Montreal, Canada, May 2007.

[7] L.-U. Axelsson, C. A. Osso, D. Cadrecha, and T. G. Johansson, "Design, performance evaluation and end wall flow structure investigation of an S-shaped intermediate turbine duct," in Proceedings of the ASME Turbo Expo, paper no. GT2007-27650, pp. 693-701, Montreal, Canada, May 2007.

[8] L.-U. Axelsson and T. G. Johansson, "Evaluation of the flow in an intermediate turbine duct at off-Design conditions," in Proceedings of the 26th Congress of International Council of the Aeronautical Sciences (ICAS '08), pp. 3286-3295, Anchorage, Alaska, USA, September 2008.

[9] A. Marn, E. Göttlich, D. Cadrecha, and H. P. Pirker, "Shorten the intermediate turbine duct length by applying an integrated concept," Journal of Turbomachinery, vol. 131, no. 4, Article ID 041014, 2009.

[10] A. D. Walker, A. G. Barker, J. F. Carrotte, J. J. Bolger, and M. J. Green, "Integrated OGV design for an aggressive s-shaped compressor transition duct," Journal of Turbomachinery, vol. 135, no. 1, Article ID 011035, 2012.

[11] E. Swain, "The design of an inlet guide vane assembly for an industrial centrifugal compressor," in IMechE Seminar on Energy Saving in the Design and Operation of Compressors, pp. 11-22, London, UK, April 1996.

[12] J. Antony, Design of Experiments for Engineers and Scientists, Butterworth-Heinemann, Oxford, UK, 2003.

[13] F. R. Menter, "Two-equation eddy-viscosity turbulence models for engineering applications," AIAA journal, vol. 32, no. 8, pp. 1598-1605, 1994.

[14] I. B. Celik, "Procedure for estimation and reporting of uncertainty due to discretization in CFD applications," Journal of Fluids Engineering, Transactions of the ASME, vol. 130, no. 7, pp. 0780011-0780014, 2008. 

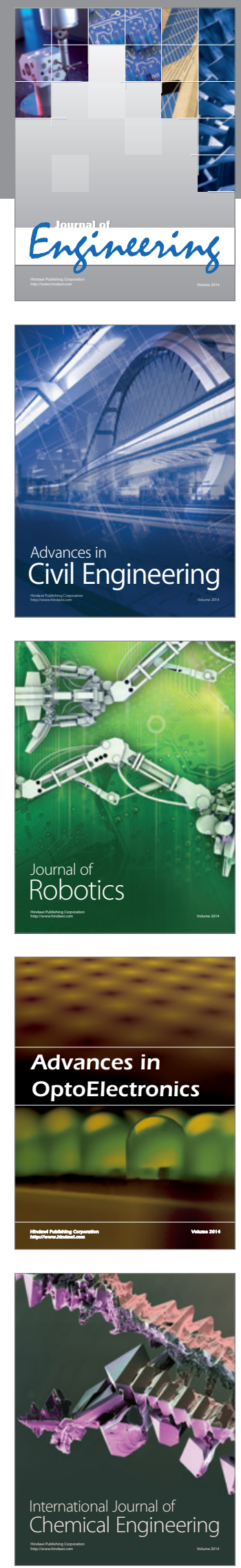

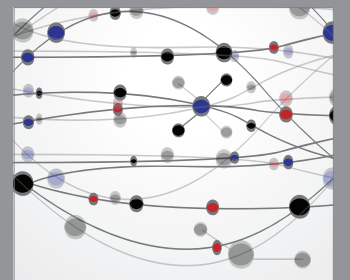

The Scientific World Journal
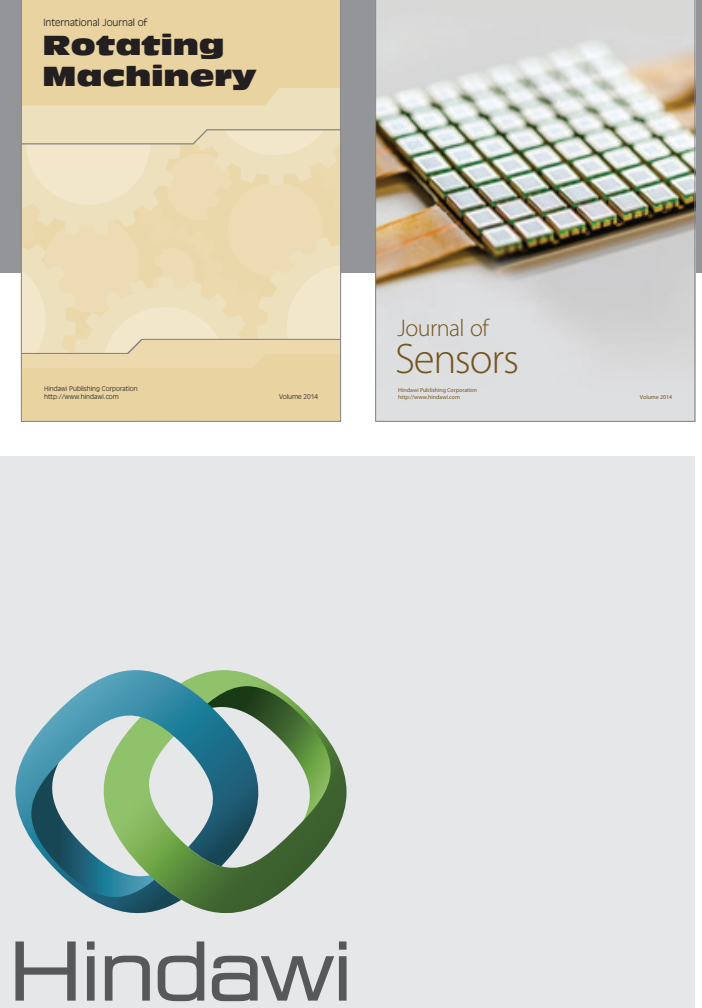

Submit your manuscripts at http://www.hindawi.com
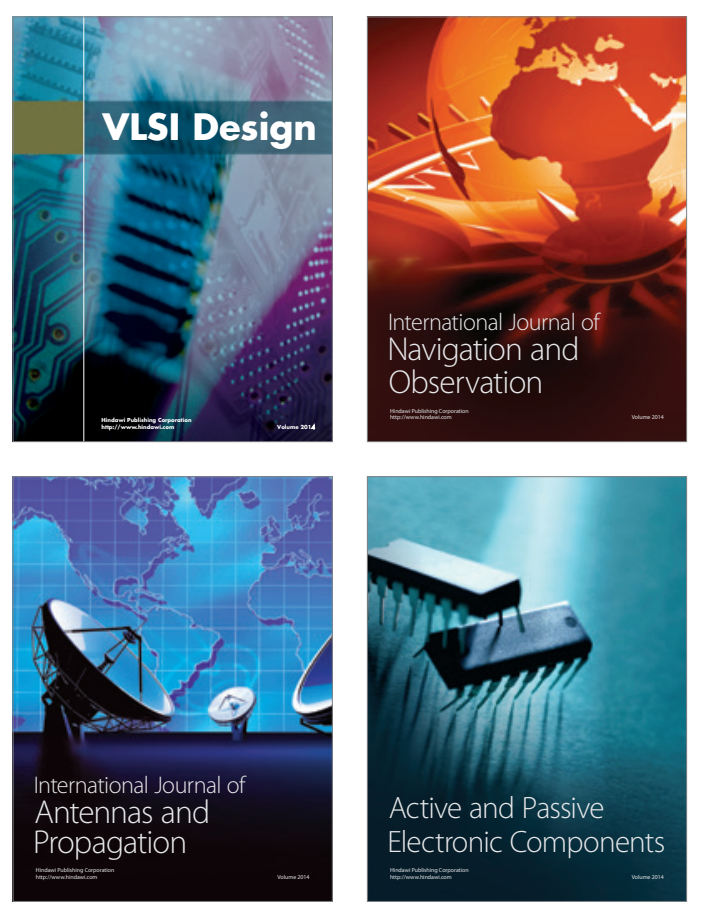
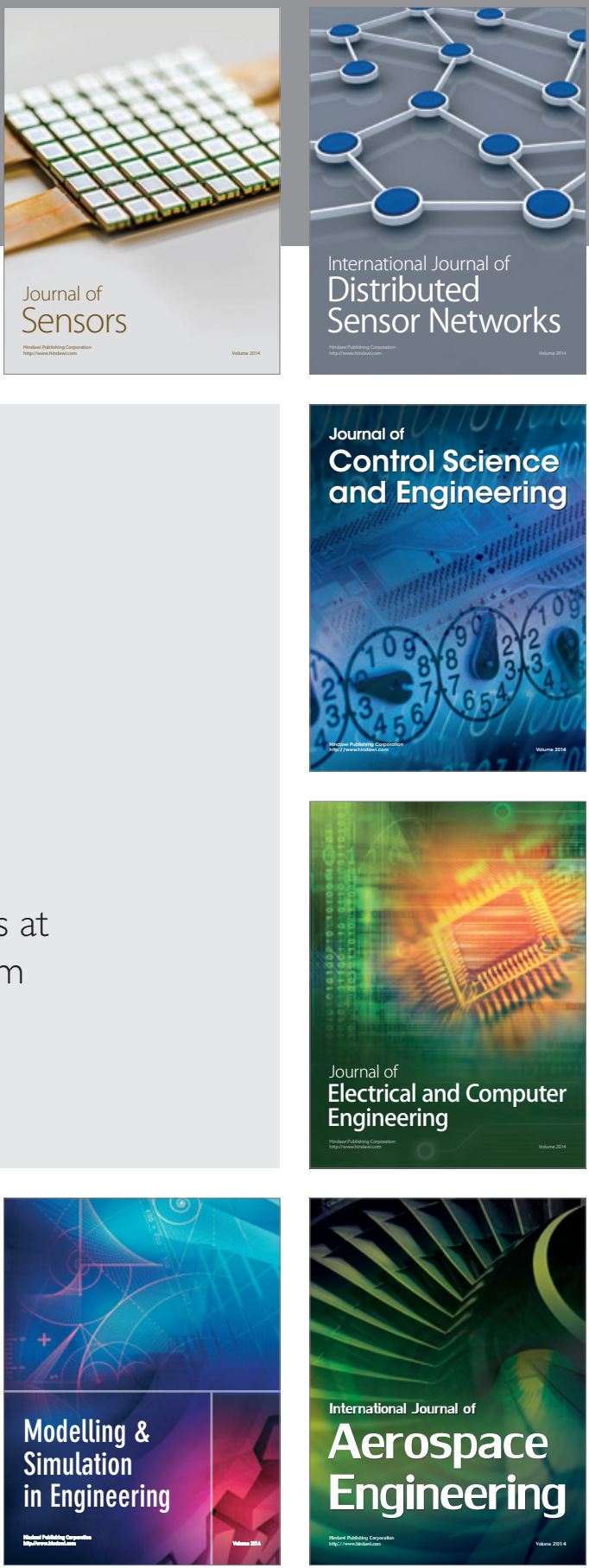

Journal of

Control Science

and Engineering
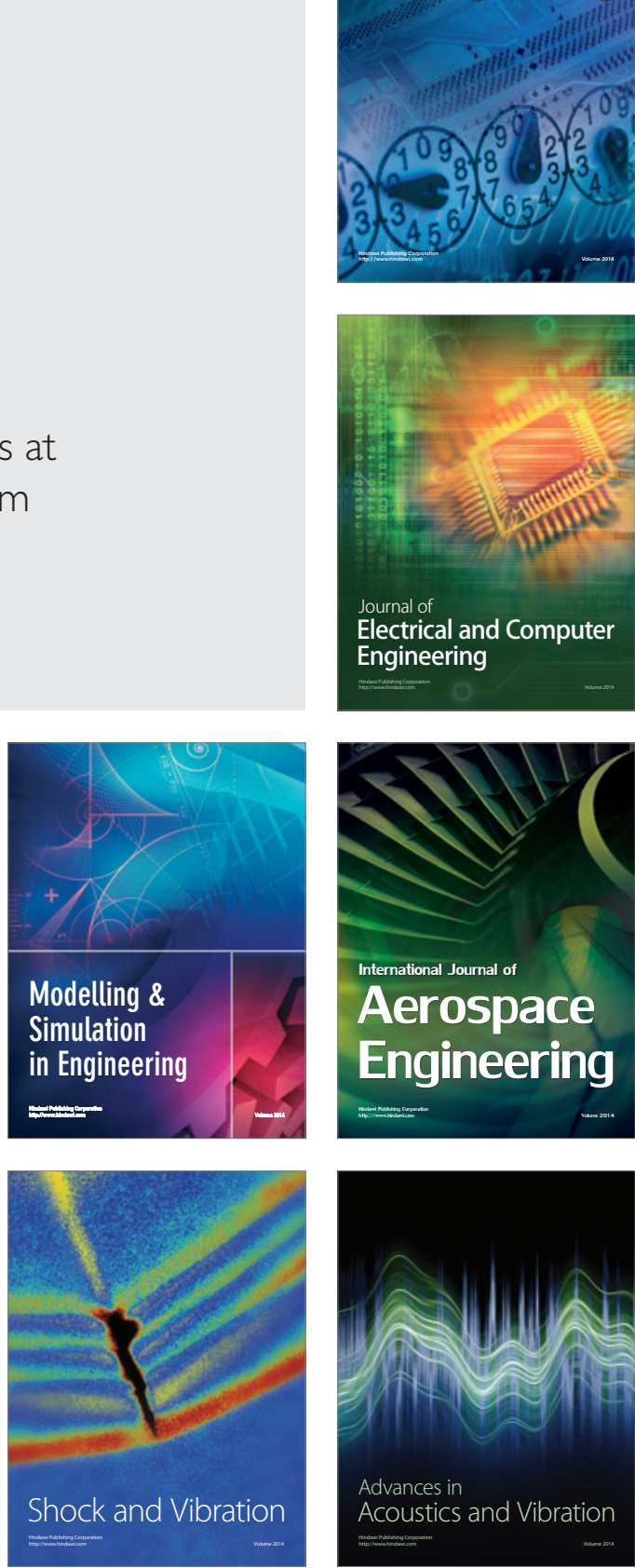